\title{
Continuous and Symmetric Trans-Capacitance Compact Model for Triple-Gate Junctionless MOSFETs
}

\author{
T.A. Oproglidis ${ }^{\mathrm{a}}$, A. Tsormpatzoglou ${ }^{\mathrm{a}}$, D.H. Tassis ${ }^{\mathrm{a}}$, C.G. Theodorou ${ }^{\mathrm{b}}$, G. Ghibaudo $^{\mathrm{b}}$, C.A. \\ Dimitriadis $^{\mathrm{a}}$ \\ ${ }^{a}$ Aristotle University of Thessaloniki, Department of Physics, 54124 Thessaloniki, Greece. \\ ${ }^{b}$ Univ. Grenoble Alpes, Univ. Savoie Mont Blanc, CNRS, Grenoble INP, IMEP-LAHC, 38000 Grenoble, France
}

\begin{abstract}
In this work, a continuous and symmetric trans-capacitance compact model for triple-gate junctionless MOSFETs is presented, valid in all regions of operation. Initially, the expressions of the gate, drain and source total charges are analytically derived based on a continuous and symmetric drain current compact model already developed. Then, the intrinsic capacitances are calculated via the differentiation of the terminal charges, verified against TCAD simulation data. The AC symmetry tests of the trans-capacitance compact model are thoroughly investigated.
\end{abstract}

Keywords: Junctionless triple-gate MOSFETs, trans-capacitance compact model, AC symmetry tests.

\section{Introduction}

Junctionless (JL) transistors have attracted much attention lately posing as candidates in the continuation of Moore's Law. Attributes provided include short-channel effects (SCE) immunity, absence of $\mathrm{p}-\mathrm{n}$ junctions that leads to a much more simplified fabrication process and improved reliability compared with the conventional MOSFETs [1].

JL transistors have recently been evaluated with regards to their analog performance. Studies have shown that JL transistors present superior analog properties, when compared to their inversion-mode (IM) counterparts and thus, their use in analog-based circuits is encouraged [2]. Generally, in order to design circuits, compact analytical models are required. These models should not only be in terms of the drain current of the respective devices, but also should describe the dynamic behavior of the devices through the inclusion of transcapacitances.

Although there are numerous compact drain current models reported for JL transistors for long-channel [3-10] and short channel devices [11-16], the literature reveals only a handful of intrinsic capacitance models. For double-gate junctionless transistors, a charge based analytical model for long-channel devices has been developed in [17]. For gate-all-around (GAA) junctionless devices, [18] is presenting an analytical model for trans-capacitances for long-channel devices whereas [19] is referring to a gate length of $100 \mathrm{~nm}$. For triple-gate JL MOSFETs, an analytical trans-capacitance model has been developed only in [20], which is described in terms of the potential at the gate interface and the potential at the buried oxide/silicon interface. It includes short channel effects (SCE) and tests the dynamic behavior of devices with gate length down to $30 \mathrm{~nm}$. However, this model requires partial derivatives of multiple intermediate variables making it complicated, whereas AC symmetry tests of the model are missing.
An attribute of the analytical models often not considered is the symmetry condition around the drain voltage $\mathrm{V}_{\mathrm{ds}}=0$ [21]. Specifically, any modern compact model should be able to succeed both the DC and AC source-drain symmetry tests. This behaviour is important for analogue and digital designs, since it dictates the suitability of the device model for a plethora of circuit applications. A few compact models have achieved the DC source-drain symmetry test for double-gate $[22,23]$ and triple-gate $[15,24]$ junctionless transistors so far. However, there is a lack of investigation on the AC symmetry of those models.

In this work, to acquire analytical expressions of the total terminal charges in triple-gate (TG) JL MOSFETs required to develop a trans-capacitance compact model, we take advantage of our DC symmetric and continuous drain current compact model in the depletion operation region [24], adequate for circuit applications of JL transistors [16]. The novel contribution of the work is to formulate an analytical and symmetric trans-capacitance compact model for short-channel triple-gate JL transistors, valid in the depletion operation region where bulk conduction determines the drain current. Its AC symmetry is validated through numerical simulation data of long and short channel devices.

\section{Trans-capacitance compact model}

The total gate charge is obtained by integrating the normalized mobile charge $\mathrm{q}_{\mathrm{y}}$ over the channel length $\mathrm{L}$ (ydirection):

$Q_{g}=-W_{e f f} C_{o x} V_{t h} \int_{0}^{L} q_{y} d y$,

where $\mathrm{W}_{\text {eff }}=\mathrm{N}_{\text {fin }}\left(2 \mathrm{H}_{\text {fin }}+\mathrm{W}_{\text {fin }}\right)$ is the effective width, $\mathrm{N}_{\text {fin }}$ is the number of fins, $\mathrm{H}_{\text {fin }}$ and $\mathrm{W}_{\text {fin }}$ are the fin height and fin width respectively, $\mathrm{L}$ is the channel length, $\mathrm{C}_{\mathrm{ox}}$ is the gate oxide capacitance per unit area and $\mathrm{V}_{\text {th }}$ is the thermal voltage. The normalized charge at coordinate $\mathrm{y}$ along the channel, $\mathrm{q}_{\mathrm{y}}=-$ $\mathrm{Q}_{\text {mobile }} /\left(2 \mathrm{C}_{\mathrm{ox}} \mathrm{V}_{\mathrm{th}} / \beta\right)$ is given by Eq. (2) in terms of the threshold voltage $V_{t}$ and the ideality factor $\eta$ defined in [24] for JL TG MOSFETs. It is noted that the parameter $\beta$ corresponds to the total capacitance reduction given by $\beta=1+\mathrm{C}_{\mathrm{ox}} \mathrm{X}_{\mathrm{dep}} / \varepsilon_{\mathrm{si}}$, where $\varepsilon_{\mathrm{si}}$ is the silicon permittivity and $\mathrm{X}_{\mathrm{dep}}$ is the depletion width which is fraction of the silicon thickness $\mathrm{t}_{\mathrm{Si}}[3]$. 


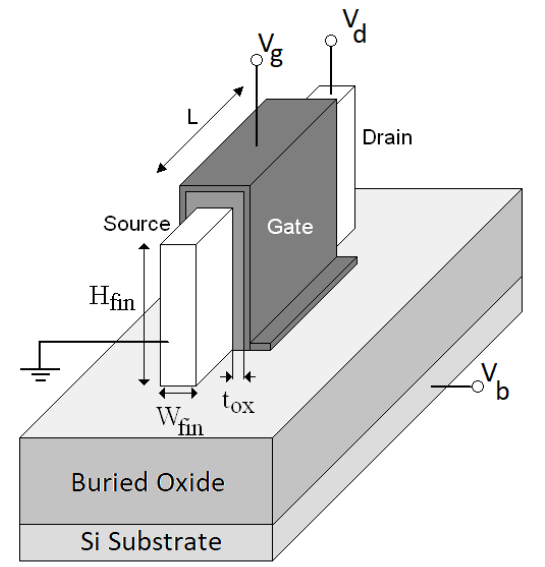

Fig. 1. Graphic representation of the simulated junctionless FinFET.

$q_{y}=\operatorname{Lambert} W\left[\exp \left(\frac{V_{g}-V_{b}-V_{t}-\eta\left(V_{y}-V_{b}\right)}{\eta V_{t h}}\right)\right]$,

where $V_{g}$ is the gate voltage and $V_{y}$ is the quasi-Fermi potential at a position $\mathrm{y}$ in the channel $\left(\mathrm{V}=\mathrm{V}_{\mathrm{s}}\right.$ at the source and $\mathrm{V}=\mathrm{V}_{\mathrm{d}}$ at the drain), referenced to the bulk potential $\mathrm{V}_{\mathrm{b}}$ of the four terminal device presented in Fig. 1. The term "four terminal device" refers to the drain current compact model of [24]. The trans-capacitance model developed in this work is practically three-terminal, ignoring the total charge of bulk, $\mathrm{Q}_{\mathrm{b}}$. In Eq. (1) dy is defined from the current continuity equation as:

$d y=-W_{e f f} \mu_{e f f} C_{o x} V_{t h} q_{y} \frac{d V_{y}}{I_{d s}(y)}$,

where $\mu_{\mathrm{eff}}$ is the effective carrier mobility. Considering the derivative of the Lambert function $\mathrm{W}(\mathrm{x})$ defined as $\mathrm{dW}(\mathrm{x}) / \mathrm{dx}=$ $\mathrm{W}(\mathrm{x}) /[\mathrm{x}(1+\mathrm{W}(\mathrm{x}))]$, from the derivative $\mathrm{dq}_{\mathrm{y}} / \mathrm{dV}_{\mathrm{y}}$ of Eq. (2) $\mathrm{dV}_{\mathrm{y}}$ is obtained as:

$d V_{y}=-\frac{V_{t h}\left(q_{y}+1\right) d q_{y}}{q_{y}}$.

Considering Eqs. (3) and (4) and the drain current defined in terms of the normalized charges at the source $\left(\mathrm{q}_{\mathrm{s}}\right)$ and drain $\left(\mathrm{q}_{\mathrm{d}}\right)[24]:$

$I_{d s}=\frac{W_{e f f} \mu_{e f f} C_{o x} V_{t h}^{2} \eta}{(1+F) L_{e f f} \beta}\left[\left(q_{s}-q_{d}\right)+\frac{1}{2}\left(q_{s}^{2}-q_{d}^{2}\right)\right]$,

the final analytical expression for the gate charge is:

$Q_{g}=A \times \frac{\frac{1}{2}\left(q_{s}+q_{d}\right)+\frac{1}{3}\left(q_{s}^{2}+q_{s} q_{d}+q_{d}^{2}\right)}{1+\frac{1}{2}\left(q_{s}+q_{d}\right)}$,

where

$$
A=\frac{W_{e f f} L_{e f f} C_{o x} V_{t h} \beta(1+F)}{\eta} .
$$

The factor $\mathrm{F}$ is defined as $\mathrm{F}=\mathrm{W}_{\mathrm{eff}} \mathrm{C}_{\mathrm{ox}} \mu_{\text {eff }} \mathrm{V}_{\mathrm{d}} \mathrm{R}_{\mathrm{sd}} / \mathrm{L}, \mathrm{R}_{\mathrm{sd}}$ is the series resistance, $\mathrm{L}_{\text {eff }}=\mathrm{L}-\Delta \mathrm{L}$ and $\Delta \mathrm{L}$ is the channel length shortening due to short-cannel effects. Both $\mu_{\text {eff }}$ and $\Delta \mathrm{L}$ are expressed in terms of the normalized charges $\mathrm{q}_{\mathrm{s}}$ and $\mathrm{q}_{\mathrm{d}}$ [24]. In JL transistors, the channel is buried in the center of the nanowire and the association of the gate oxide capacitance per unit area $\mathrm{C}_{\mathrm{ox}}$ with the depletion capacitance $\mathrm{C}_{\mathrm{dep}}$ due to the depletion formed between the gate oxide and the mobile charges, reduces the total capacitance that drives the drain current. The normalized charges $\mathrm{q}_{\mathrm{s}}$ and $\mathrm{q}_{\mathrm{d}}$ are obtained from Eq. (2) with $\mathrm{V}_{\mathrm{y}}=0$ at the source $(\mathrm{y}=0)$ and $\mathrm{V}_{\mathrm{y}}=\mathrm{V}_{\mathrm{d}}$ at the drain $(\mathrm{y}=\mathrm{L})$. We note that the velocity saturation is incorporated into the factor $\mathrm{F}$ through the effective carrier mobility.

Following the Ward-Dutton channel charge-partitioning scheme [25], the total charge at the drain is obtained by integrating the normalized mobile charge $\mathrm{q}_{\mathrm{y}}$ over the channel length $\mathrm{L}$ (y-direction) as:

$Q_{d}=-W_{e f f} C_{o x} V_{t h} \int_{0}^{L} \frac{y}{L} q_{y} d y$.

Due to the current continuity requiring constant drain current at any point $\mathrm{y}$ of the channel, considering that $\mathrm{I}_{\mathrm{ds}}(\mathrm{y})=\mathrm{I}_{\mathrm{ds}}(\mathrm{y}=\mathrm{L})$ and using the proportional partitioning method, for constant mobility in the channel direction y can be expressed by:

$\frac{y}{L}=\frac{\left(q_{s}-q_{y}\right)+\frac{1}{2}\left(q_{s}^{2}-q_{y}^{2}\right)}{\left(q_{s}-q_{d}\right)+\frac{1}{2}\left(q_{s}^{2}-q_{d}^{2}\right)}$.

By introducing Eqs. (3) and (9) in Eq. (8), the analytical expression for the drain charge is:

$Q_{d}=-\frac{A}{\left[1+\frac{1}{2}\left(q_{s}+q_{d}\right)\right]^{2}} \cdot\left(\varphi_{1}+\varphi_{2}+\varphi_{3}\right)$,

(10)

where

$\varphi_{1}=\frac{1}{6} q_{s}+\frac{5}{24} q_{s}^{2}+\frac{1}{15} q_{s}^{3}$,

$\varphi_{2}=\frac{1}{3} q_{d}+\frac{3}{8} q_{d}^{2}+\frac{1}{10} q_{d}^{3}$

$\varphi_{3}=q_{s} q_{d}\left(\frac{5}{12}+\frac{2}{15} q_{s}+\frac{1}{5} q_{d}\right)$.

Considering the charge conservation equation, the total charge at the source is given by the relation:

$Q_{s}=-Q_{g}-Q_{d}=-A \times \frac{\frac{1}{2}\left(q_{s}+q_{d}\right)+\frac{1}{3}\left(q_{s}^{2}+q_{s} q_{d}+q_{d}^{2}\right)}{1+\frac{1}{2}\left(q_{s}+q_{d}\right)}+$ 


$$
A \times \frac{\left(\varphi_{1}+\varphi_{2}+\varphi_{3}\right)}{\left[1+\frac{1}{2}\left(q_{s}+q_{d}\right)\right]^{2}} .
$$

It is noted that the terminal charges Eqs. (6) and (10) are continuous at $\mathrm{V}_{\mathrm{ds}}=0$.

The analytical equations of the total charges at the gate, drain and source electrodes are generally complex for every trans-capacitance model developed in literature. This feature is also present here in Eqs. (6), (10) and (14). However, these analytical equations can become quite compact in the linear $\left(q_{s} \square q_{d}\right)$ and saturation $\left(q_{d} \square 0\right)$ regions. Therefore, for the total gate and drain charges in the linear region, Eqs. (6) and (10) are simplified as:

$$
\begin{aligned}
& Q_{g, \text { lin }}=A \cdot q_{s}=A \cdot q_{d}, \\
& Q_{\mathrm{d}, \text { lin }}=\frac{A \cdot q_{s} \cdot\left(1+q_{s}\right)}{2}=\frac{A \cdot q_{d} \cdot\left(1+q_{d}\right)}{2},
\end{aligned}
$$

For the saturation region, Eqs. (6) and (10) can be written as:

$$
\begin{aligned}
Q_{g, \text { sat }} & =\frac{A \cdot q_{s}}{3} \cdot\left(\frac{2 q_{s}+3}{q_{s}+2}\right), \\
Q_{\mathrm{d}, \mathrm{sat}} & =\frac{A \cdot q_{s}}{30\left(q_{s}+2\right)^{2}} \cdot\left(8 q_{s}^{2}+25 q_{s}+20\right) .
\end{aligned}
$$

It is apparent that the total source charge in the linear $\left(\mathrm{Q}_{\mathrm{s}, \mathrm{lin}}\right)$ and saturation $\left(\mathrm{Q}_{\mathrm{s}, \mathrm{sat}}\right)$ regions can be analytically obtained by adding Eqs. (15), (16) and (17), (18) respectively.

Parasitic capacitance $\left(\mathrm{C}_{\mathrm{p}}\right.$ in $\left.\mathrm{F} / \mu \mathrm{m}\right)$, including overlap and fringe capacitances must be considered as a serious problem on trans-capacitances [10]. Taking into account $C_{p}$, then the total terminal charges of the gate, drain and source can be, respectively, expressed as:

$$
\begin{aligned}
& Q_{t, g}=Q_{g}+C_{p} W_{e f f} V_{g s}+C_{p} W_{e f f} V_{g d}, \\
& Q_{t, d}=Q_{d}-C_{p} W_{e f f} V_{g d}, \\
& Q_{t, s}=Q_{s}-C_{p} W_{e f f} V_{g s} .
\end{aligned}
$$

Having acquired the analytical expressions of the total charges for gate, source and drain, the intrinsic capacitances can be obtained from Eq. (22):

$$
C_{i j}=-\left.\frac{\partial Q_{t, i}}{\partial V_{j}}\right|_{i \neq j}, C_{i j}=\left.\frac{\partial Q_{t, i}}{\partial V_{j}}\right|_{i=j},
$$

where $i$ and $j$ stand for the source, drain and gate.

\section{Trans-capacitance model validation}

To verify the accuracy of the trans-capacitance compact model, TCAD numerical simulations were performed using Silvaco (Atlas) tool, considering the drift-diffusion transport mechanism. In simulations, models for the bandgap narrowing, Shockley-Read-Hall generation-recombination and fielddependent mobility have been used. The simulated device has channel length $\mathrm{L}=1 \mu \mathrm{m}$ and $30 \mathrm{~nm}$, fin height $\mathrm{H}_{\text {fin }}=30 \mathrm{~nm}$, fin width $\mathrm{W}_{\text {fin }}=10 \mathrm{~nm}$, equivalent gate oxide thickness $\mathrm{t}_{\mathrm{ox}}=$ $1.3 \mathrm{~nm}$ and doping concentration $\mathrm{N}_{\mathrm{d}}=6 \times 10^{18} \mathrm{~cm}^{-3}$. The source/drain regions used for simulations were short to reduce the effect of parasitic resistances. Quantum confinement effects are not considered here, since these effects become significant when the silicon thickness is smaller than $10 \mathrm{~nm}$ [25].

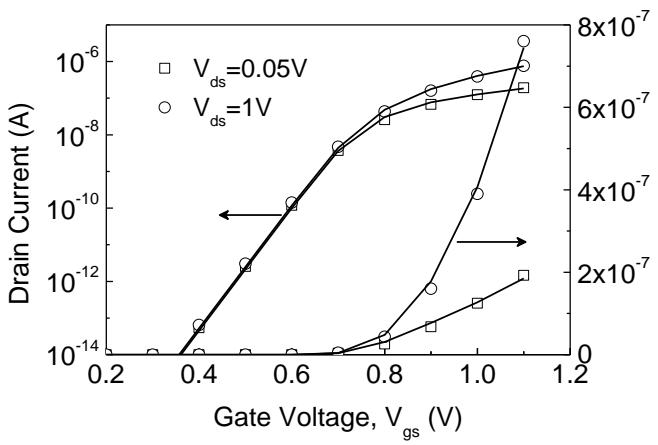

Fig. 2. Transfer characteristics of a long-channel $(L=1 \mu \mathrm{m})$ TG JL MOSFET with different drain voltages $\mathrm{V}_{\mathrm{ds}}$ in logarithmic and linear scales. Symbols and lines correspond to TCAD simulation and model results, respectively. The model results were obtained using the parameters: $\eta=1.01, \mathrm{~V}_{\mathrm{t}}=0.713 \mathrm{~V}$, $\mu=145 \mathrm{~cm}^{2} / \mathrm{Vs}$ and series resistance $\mathrm{R}_{\mathrm{sd}}=3 \mathrm{k} \Omega$.
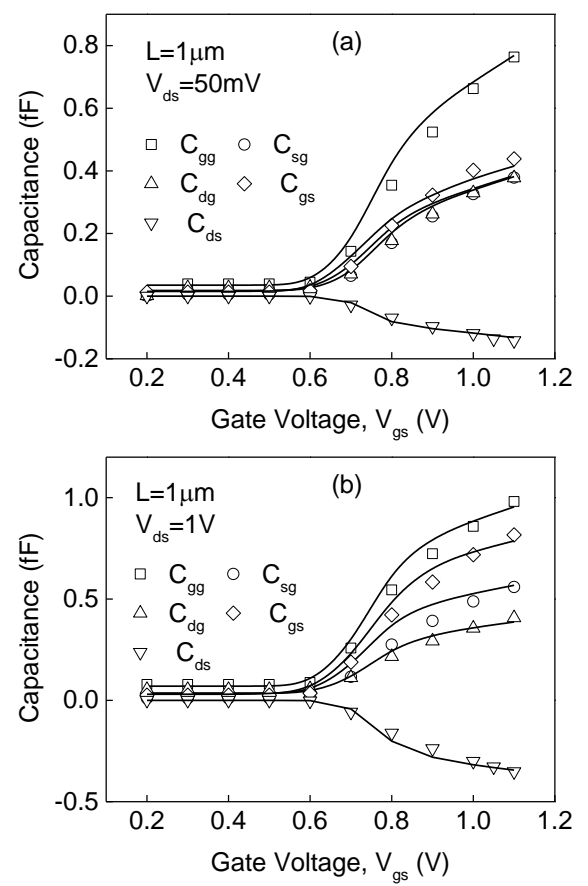

Fig. 3. Gate voltage dependence of trans-capacitances for a long-channel $(\mathrm{L}=$ $1 \mu \mathrm{m})$ TG JL MOSFET at drain voltages $\mathrm{V}_{\mathrm{ds}}=50 \mathrm{mV}$ (a) and $1 \mathrm{~V}$ (b) in the depletion operation region. Symbols and lines correspond to TCAD simulation and model results, respectively. The model results were obtained using the parameters of the drain current model and the parasitic capacitance $\mathrm{C}_{\mathrm{p}}=5 \times 10^{-12} \mathrm{~F} / \mu \mathrm{m}$. 
Figs. 2 and 3 show the transfer characteristics in logarithmic and linear scales ant the trans-capacitances of a long-channel $(\mathrm{L}=1 \mu \mathrm{m}) \mathrm{JL}$ TG MOSFET at $\mathrm{V}_{\mathrm{ds}}=0.05$ and $1 \mathrm{~V}$. In order to acquire the best possible results, an optimization technique was performed to fit the transfer characteristics and the transcapacitances models simultaneously to the TCAD data at drain voltages 0.05 and $1 \mathrm{~V}$.

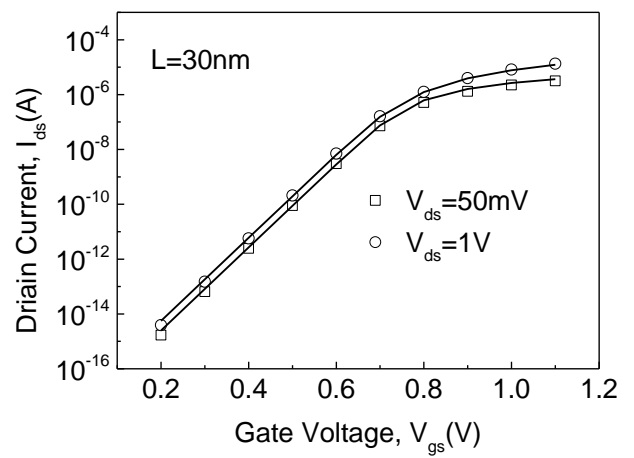

Fig. 4. Transfer characteristics of a short-channel $(\mathrm{L}=30 \mathrm{~nm})$ TG JL MOSFET with different drain voltages $\mathrm{V}_{\mathrm{ds}}$ in logarithmic and linear scales. Symbols and lines correspond to TCAD simulation and model results, respectively. The model results were obtained using the parameters: Ideality $\eta$ $=1.01, \mathrm{~V}_{\mathrm{t}}=0.7 \mathrm{~V}, \mu=98.2 \mathrm{~cm}^{2} / \mathrm{Vs}$, series resistance $\mathrm{R}_{\mathrm{sd}}=2.87 \mathrm{k} \Omega$ and channel length modulation parameter $\delta=0.121 \mathrm{~V}^{-2}$ [24].
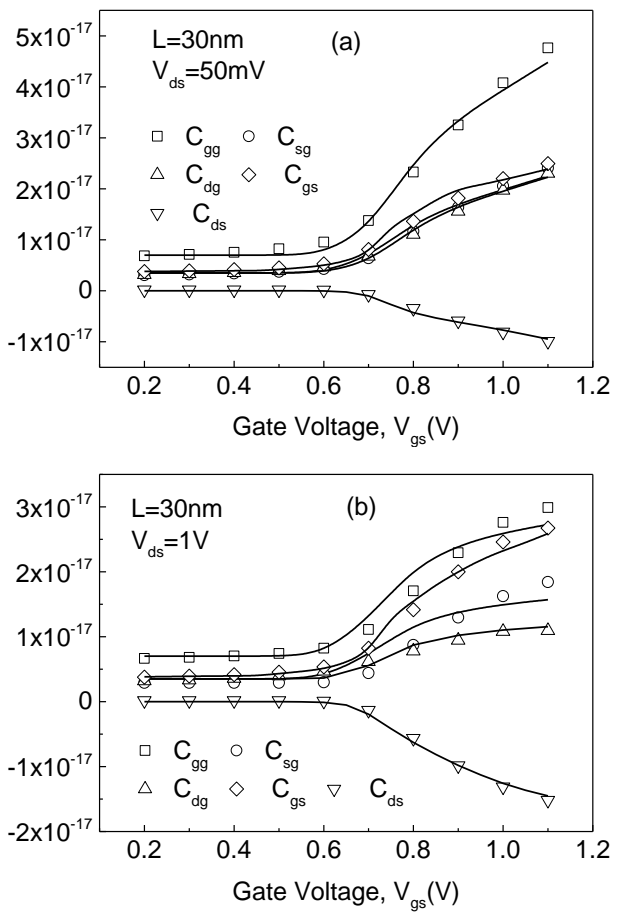

Fig. 5. Gate voltage dependence of trans-capacitances for a short-channel $(\mathrm{L}=$ $30 \mathrm{~nm}$ ) TG JL MOSFET at drain voltages $\mathrm{V}_{\mathrm{ds}}=50 \mathrm{mV}$ (a) and $1 \mathrm{~V}$ (b) in the depletion operation region. Symbols and lines correspond to TCAD simulation and model results, respectively. The model results were obtained using the parameters of the drain current model and the parasitic capacitance $\mathrm{C}_{\mathrm{p}}=5 \times 10^{-13} \mathrm{~F} / \mu \mathrm{m}$.

The results of the symmetric drain current compact model [24] are in good agreement with the Silvaco simulation data. The model parameters are presented in the caption of Fig. 2. Fig. 3 shows the trans-capacitances obtained from numerical simulations and the proposed analytical compact model using a parasitic capacitance $\mathrm{C}_{\mathrm{p}}=5 \times 10^{-12} \mathrm{~F} / \mu \mathrm{m}$. All the modeled trans-capacitances show good agreement with the simulated data, indicating the good accuracy of the proposed transcapacitance compact model. It is noticed that the models results for both drain current and trans-capacitances fit the simulation data for depletion width $\mathrm{X}_{\mathrm{dep}}=\mathrm{t}_{\mathrm{Si}} / 7$ in the model parameter $\beta$.

Figs. 4 and 5 present the transfer characteristics and the transcapacitances of a short-channel $(\mathrm{L}=30 \mathrm{~nm}) \mathrm{JL}$ TG MOSFET for different drain voltages. Good agreement between numerical and model results has been obtained indicating that the charge-based trans-capacitance model is valid for shortchannel JL TG MOSFETs in the depletion operation region. Using the capacitances presented in Figs. 3 and 5, all other capacitances can be obtained by means of the following relationships derived according to the charge conservation law: $\mathrm{C}_{\mathrm{ss}}=\mathrm{C}_{\mathrm{ds}}+\mathrm{C}_{\mathrm{gs}}, \mathrm{C}_{\mathrm{dd}}=\mathrm{C}_{\mathrm{ds}}+\mathrm{C}_{\mathrm{dg}}, \mathrm{C}_{\mathrm{gd}}=\mathrm{C}_{\mathrm{gg}}-\mathrm{C}_{\mathrm{gs}}, \mathrm{C}_{\mathrm{sd}}=\mathrm{C}_{\mathrm{dd}}+$ $\mathrm{C}_{\mathrm{gd}}$.

\section{AC symmetry test}

In addition to the drain current symmetry [24], the terminal charges and, therefore, the trans-capacitances should also satisfy the symmetry condition. According to [21], AC symmetry tests of the gate and source/drain charge models were performed by calculating the quantities $\delta_{\mathrm{cg}}$ and $\delta_{\mathrm{csd}}$, respectively, defined as:

$\delta_{c g}=\frac{C_{g s}-C_{g d}}{C_{g s}+C_{g d}}$

and

$\delta_{c s d}=\frac{C_{s s}-C_{d d}}{C_{s s}+C_{d d}}$.

The trans-capacitance compact model succeeds in the AC symmetry test when Eqs. (23) and (24) are odd functions of $\mathrm{V}_{\mathrm{x}}$, which is a voltage that is generally given low values. In this work, the drain voltage is rendered equal to $V_{x}$ and the source voltage equal to $-\mathrm{V}_{\mathrm{x}}$. Then $\mathrm{V}_{\mathrm{x}}$ is swept from -0.2 to 0.2 $\mathrm{V}$, while the gate voltage $\mathrm{V}_{\mathrm{g}}$ maintains a constant value. In Figs. 6 and 7, we show that the model satisfies the condition for AC gate and source-drain symmetry, respectively, for typical long-channel TG JL transistor with parameters: $\mathrm{L}=1$ $\mu \mathrm{m}, \mathrm{H}_{\text {fin }}=30 \mathrm{~nm}, \mathrm{~W}_{\text {fin }}=10 \mathrm{~nm}, \mathrm{t}_{\mathrm{ox}}=1 \mathrm{~nm}$ and $\mathrm{N}_{\mathrm{d}}=6 \times 10^{18}$ $\mathrm{cm}^{-3}$. In these figures, we also show the first derivatives $\mathrm{d} \delta_{\mathrm{cg}} / \mathrm{dV}_{\mathrm{x}}$ and $\mathrm{d} \delta_{\mathrm{csd}} / \mathrm{dV}_{\mathrm{x}}$, which highlight the smoothness and continuity of our trans-capacitance compact model. Symmetry and continuity of the second order derivatives $d \delta_{c g}^{2} / d V_{x}^{2}$ and $d \delta_{c s d}^{2} / d V_{x}^{2}$ is confirmed in Fig. 8 for a long-channel devices $(\mathrm{L}=1 \mu \mathrm{m})$ at $\mathrm{V}_{\mathrm{ds}}=0$ and $1 \mathrm{~V}$, as it was also confirmed for higher order derivatives. The symmetry and continuity of $d \delta_{c g}^{2} / d V_{x}^{2}$ and $d \delta_{c s d}^{2} / d V_{x}^{2}$ for short-channel device $(\mathrm{L}=30$ $\mathrm{nm})$ for $\mathrm{V}_{\mathrm{ds}}=0$ and $1 \mathrm{~V}$ is confirmed in Fig. 9. 

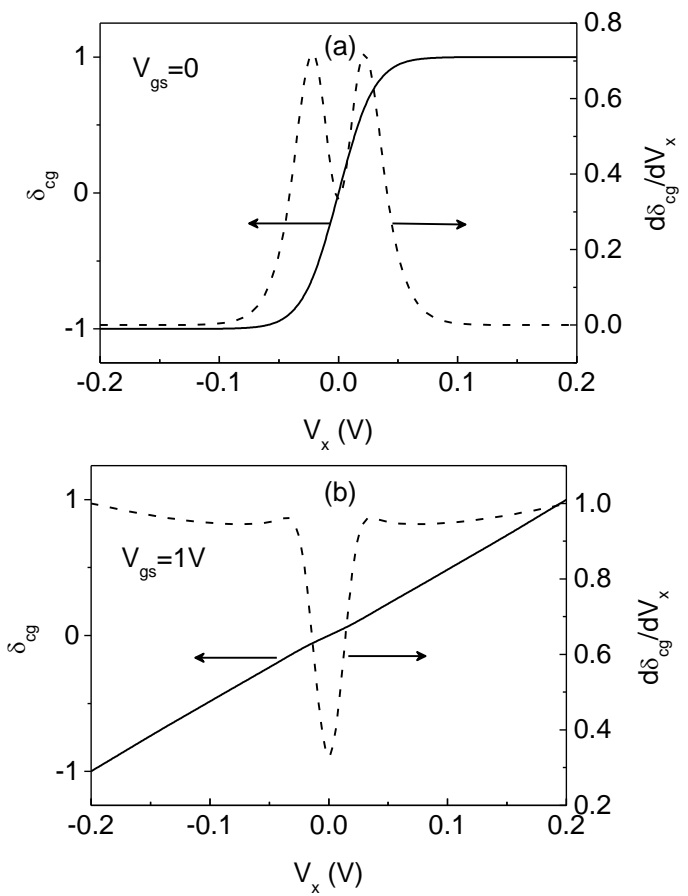

Fig. 6. AC symmetry test $\delta_{\mathrm{cg}}$ of the gate charge and its first derivative versus $\mathrm{V}_{\mathrm{x}}$ for (a) $\mathrm{V}_{\mathrm{g}}=0$ and (b) $\mathrm{V}_{\mathrm{g}}=1 \mathrm{~V}$ of a long-channel $(\mathrm{L}=1 \mu \mathrm{m}) \mathrm{TG} \mathrm{JL}$ MOSFET. $\delta_{\mathrm{cg}}$ and its derivative have been normalized to their absolute maximum values.
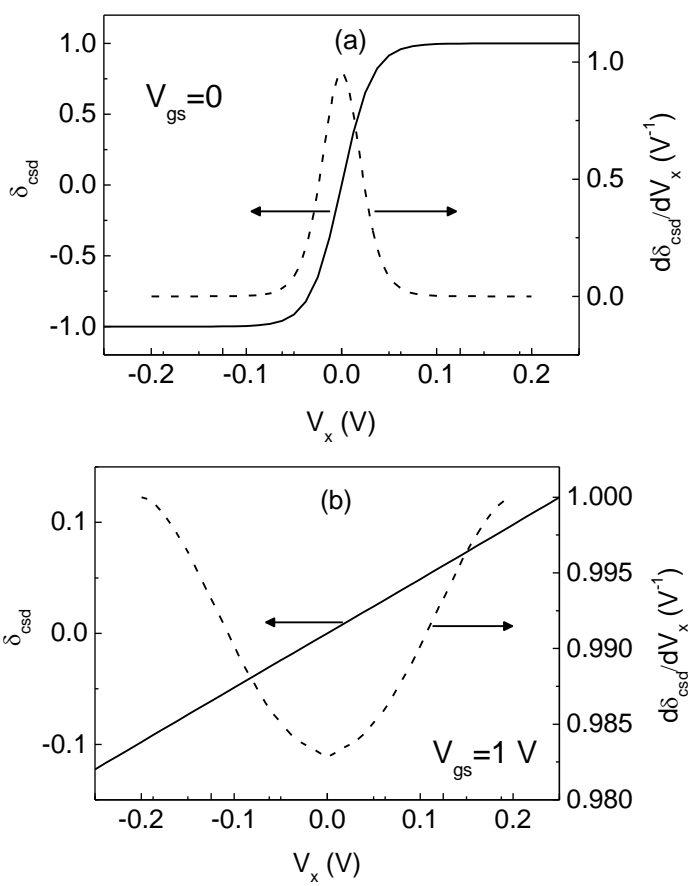

Fig. 7. AC symmetry test $\delta_{\text {csd }}$ of the source/drain charge and its first derivative versus $V_{x}$ for (a) $V_{g}=0$ and (b) $V_{g}=1 \mathrm{~V}$ of a long-channel $(L=1$ $\mu \mathrm{m})$ TG JL MOSFET $\delta_{\mathrm{csd}}$ and its derivative have been normalized to their absolute maximum values.
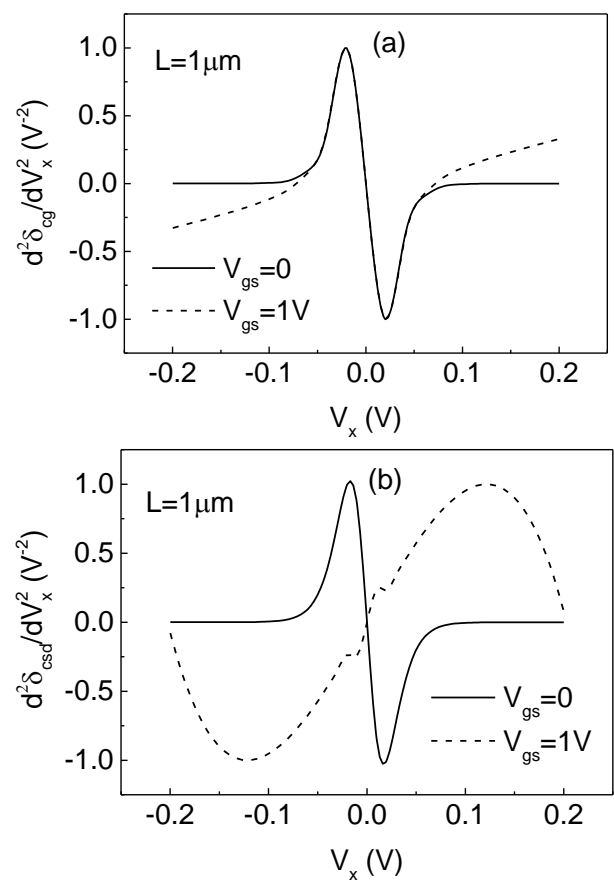

Fig. 8. Second order derivatives of AC symmetry test $d \delta_{c g}^{2} / d V_{x}^{2}$ (a) and $d \delta_{c s d}^{2} / d V_{x}^{2}(\mathrm{~b})$ versus $\mathrm{V}_{\mathrm{x}}$ for $\mathrm{V}_{\mathrm{g}}=0$ and $1 \mathrm{~V}$ of a long-channel $(\mathrm{L}=1 \mu \mathrm{m})$ TG JL MOSFET. The derivatives have been normalized to their absolute maximum values.
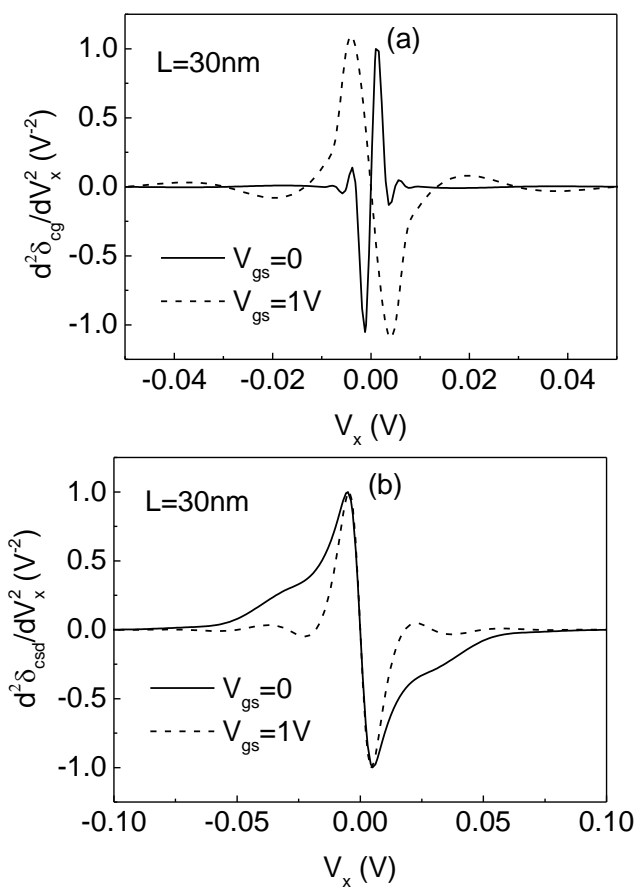

Fig. 9. Second order derivatives of AC symmetry test $d \delta_{c g}^{2} / d V_{x}^{2}$ (a) and $d \delta_{c s d}^{2} / d V_{x}^{2}(\mathrm{~b})$ versus $\mathrm{V}_{\mathrm{x}}$ for $\mathrm{V}_{\mathrm{g}}=0$ and $1 \mathrm{~V}$ of a short-channel $(\mathrm{L}=30 \mathrm{~nm})$ TG JL MOSFET. The derivatives have been normalized to their absolute maximum values. 


\section{Conclusion}

A physics-based compact model for trans-capacitances has been developed in this work for long-channel and shortchannel TG JL MOSFETs, valid in all regions of operation. The trans-capacitances model was derived based on the Dutton-Ward channel linear charge partition method and its development was thoroughly described. The model has been validated against TCAD data, demonstrating good agreement. AC symmetry tests were performed in order to verify the continuous and symmetric nature of the trans-capacitance compact model. The model is shown to pass the AC symmetry condition tests and thus the compact trans-capacitance model developed seems suitable for implementation in circuit design simulators.

\section{Acknowledgement}

This research is co-financed by Greece and the European Union (European Social Fund-ESF) through the Operational Programme «Human Resources Development, Education and Lifelong Learning» in the context of the project "Strengthening Human Resources Research Potential via Doctorate Research" (MIS-5000432), implemented by the State Scholarships Foundation (IKY).

\section{References}

[1] Colinge J.-P. et al.. Nanowire transistors without junctions. Nature Nanotechnol.2010;5(3):225-9.

[2] Doria RT et al., Junctionless multiple-gate transistors for analog applications. IEEE Trans. Electron Devices 2011;58(8):2511-19.

[3] Duarte JP, Choi SJ, Choi YK. A full-range drain current model for double-gate junctionless transistors. IEEE Trans. Electron Devices 2011;58(12):4219-25.

[4] Duarte JP, Choi SJ, Moon DI, Choi YK. Simple analytical bulk current model for long-channel double-gate junctionless transistors. IEEE Electron Device Lett. 2011;32(6):704-706.

[5] Chen $Z$ et al., Surface-potential-based drain current model for longchannel junctionless double-gate MOSFETs. IEEE Trans. Electron Devices 2012;59(12):3292-98.

[6] Trevisoli RD, Doria RT, De Souza M, Das S, Ferain I, Pavanello MA. Surface-potential-based drain current analytical model for triple-gate junctionless nanowire transistors. IEEE Trans. Electron Devices 2012; 59(12):3510-18, 2012.

[7] Jazaeri F, Barbut L, Koukab A, Sallese JM. Analytical model for ultrathin body junctionless symmetric double gate MOSFETs in subthreshold regime. Solid-State Electron. 2013;82:103-110.

[8] Cerdeira A, Avila F, Iniguez B, De Souza M, Pavanello MA, Estrada M. Compact core model for symmetric double-gate junctionless transistors," Solid-State Electron. 2014;94:91-7.

[9] Lin X, Zhang B, Xiao Y, Lou H, Zhang L, Chan M. Analytical current model for long-channel junctionless double-gate MOSFETs. IEEE Trans. Electron Devices 2016;63(3):959-65.

[10] Jiang C, Liang R, Xu J, Alam MA. A compact quasi-static terminal charge and drain current model for double-gate junctionless transistors and its circuit validation. IEEE Trans. Electron Devices 2017;64(12):4823-30.

[11] Hwang BW, Yang JW, Lee SH. Explicit analytical current-voltage model for double-gate junctionless transistors. IEEE Trans. Electron Devices.2015;62(1):171-7.

[12] Holtij T, Kloes A, Iniquez B. 3-D compact model for nanoscale junctionless triple-gate nanowire MOSFETs, including simple treatment of quantization effects. Solid-State Electron. 2015;112, 8598.
[13] Baruah RK, Paily RP. A surface-potential based drain current model for short-channel symmetric double-gate junctionless transistor. J. Comput. Electron. 2016;15(1):45-52.

[14] Holtij T, Graef M, Hain FM, Kloes A, Iniguez B. Compact model for short-channel junctionless accumulation mode double gate MOSFETs. IEEE Trans. Electron Devices 2014;61(2):288-99.

[15] Avila-Herrera F, Paz BC, Cerdeira A, Estrada M, Pavanello MA. Charge-based compact analytical model for triple gate junctionless nanowire transistors. Solid-State Electron. 2016;122:23-31.

[16] Oproglidis TA et al.. Analytical drain current compact model in the depletion operation region of short-channel triple-gate junctionless transistors. IEEE Trans. Electron Devices 2017;64(1):66-72.

[17] Jazaeri F, Barbut L, Sallese JM. Trans-capacitance modeling in junctionless symmetric double-gate MOSFETs. IEEE Trans. Electron Devices 2013;60(12):4034-40.

[18] Moldovan O, Lime F, Iniguez B. A compact explicit model for longchannel gate-all-around junctionless MOSFETs. Part II: Total charges and intrinsic capacitance characteristics. IEEE Trans. Electron Devices 2014;61(9):3042-6, 2014.

[19] Jazaeri F, Barbut L, Sallese JM. Trans-capacitance modeling in junctionless gate-all-around nanowire FETs. Solid-State Electron. 2014; 96:34-7.

[20] Trevisoli R, Doria RT, De Souza M, Barraud S, Vinet M, Pavanello MA. Analytical model for the dynamic behavior of triple-gate junctionless nanowire transistors. IEEE Trans. Electron Devices 2016; 63(2): 856-63.

[21] McAndrew CC. Validation of MOSFET model source-drain symmetry. IEEE Trans. Electron Devices 2006;53(9):2202-6.

[22] Jiang C, Liang R, Xu J, Alam MA. A compact quasi-static terminal charge and drain current model for double-gate junctionless transistors and its circuit validation. IEEE Trans. Electron Devices 2017;64(12): 4823-30.

[23] Holtij T, Graef M, Hain FM, Kloes A, Iniguez B. Compact model for short-channel junctionless accumulation mode double gate MOSFETs. IEEE Trans. Electron Devices 2013;61(2):288-99.

[24] Oproglidis TA, Tsormpatzoglou A, Theodorou CG, Karatsori TA, Ghibaudo G. Dimitriadis CA. Upgrade of drain current compact model for nanoscale triple-gate junctionless transistors to continuous and symmetric. IEEE Trans. Electron Devices 2019;66(10):4486-9.

[25] A. Yesayan, F. Pregaldiny, N. Chevillon, C. Lallement, and J.-M. Sallese, "Physics-based compact model for ultra-scaled FinFETs," Solid-State Electron., vol. 62, pp. 165-173, 2011.

[26] Ward DE, Dutton RW. A charge-oriented model for MOS transistor capacitances. IEEE J. Solid-State Circuits 1978;13(5):703-8. 